\title{
A study on exercise habits of Japanese mothers with preschool children: the differences in the amounts of exercise and exercise self-efficacy by the duration of exercise play with children
}

Chihiro KEMURIYAMA (Gifu Shotoku Gakuen University, Japan), Mitsuhiro AMAZAKI (Aichi University, Japan)

Keywords: Japanese mothers, preschool children, exercise play, physical activity amounts, self-efficacy

\section{Background}

Mental Health of Japanese

mothers with preschool children

$\checkmark$ Japanese mothers with pre-school children are under stress from child-rearing such as the rapid development of childrens'

physical and mental, expansion of range of behavior, language development

(Hasegawa, 2014).

$\checkmark$ Japanese mothers with high parenting stress are less responsive in mother-child interaction negotiation scenes than low mothers (Hasegawa, 2008).

$\checkmark$ The mental health status of mothers who have young children exerts a maio influence on the growth of their children. Therefore, it is important for mothers to maintain their own well-being (Ozeki, et al., 2014).

\section{The effect of mother's physical} activity (PA)

$\checkmark$ Regular exercise is assists in relieving stress, reducing depression, improving mood, and alleviating negative emotions.

$\checkmark$ Exercise play done by mother and children is not only to encourage children's development and interact with each other by mother and children, but also to eliminate exercise shortage and stress for mother (Motonaga, 2014). However, Japanese women with young children are less likely to engage in adequate exercise than women without children.

$\checkmark$ Self-efficacy is an individual's belief in performing an action, and if a person's sense of self-efficacy in an activity is high, the frequency of undertaking such actions also increases.

\section{Purpose}

The purpose of this study is to examine the differences in the amounts of exercise and the exercise self-efficacy scores of mothers with preschoo children by the duration of exercise play with the children.

\section{Method}

1. Participants

504 Japanese mothers with preschool children $(\mathrm{M}$ age $=34.32$ years, $S D=$ 4.516)

2. Measures

1) Face sheet: sex, age

2) Log of the duration of the exercise play with her children each day

3) Kasari's PA Index is a valid measure of the amount of PA (frequency $x$ intensity $x$ time)

4) Exercise Self-efficacy Scale

3. Analysis

Independent sample t-tests were conducted to investigate the differences in the amounts of PA and exercise self-efficacy by the duration of exercise play with their children.

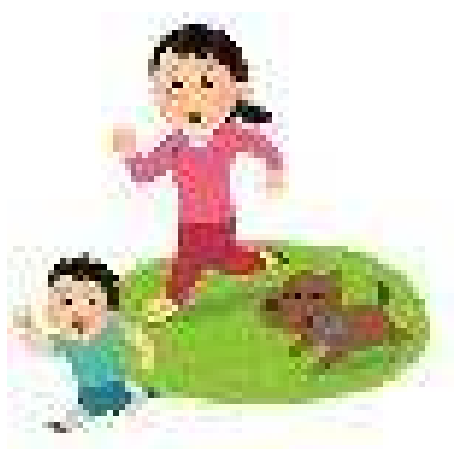

\section{Results and Conclusion}

The results of the t-tests revealed that the scores of PA amounts $(t[502]=-1.830, p$ $<.10, d=.16)$, recovery self-efficacy $(t[502]=-2.767, p<.01, d=.25)$ and coping selfefficacy $(t[502]=-2.575, p<.05, d=.23)$ were significantly higher in mothers who were the long-duration exercise play group than the short-duration exercise play group.
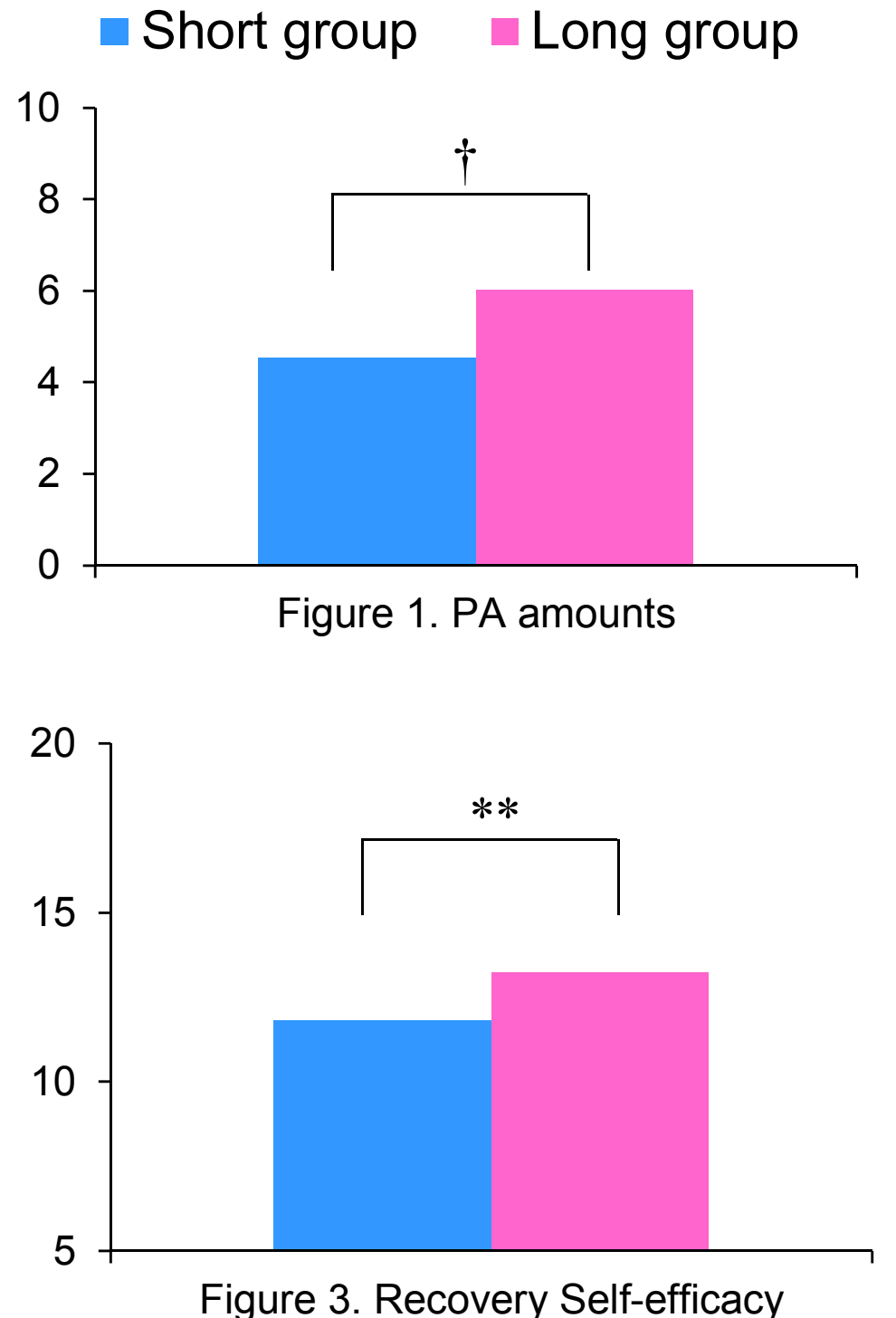
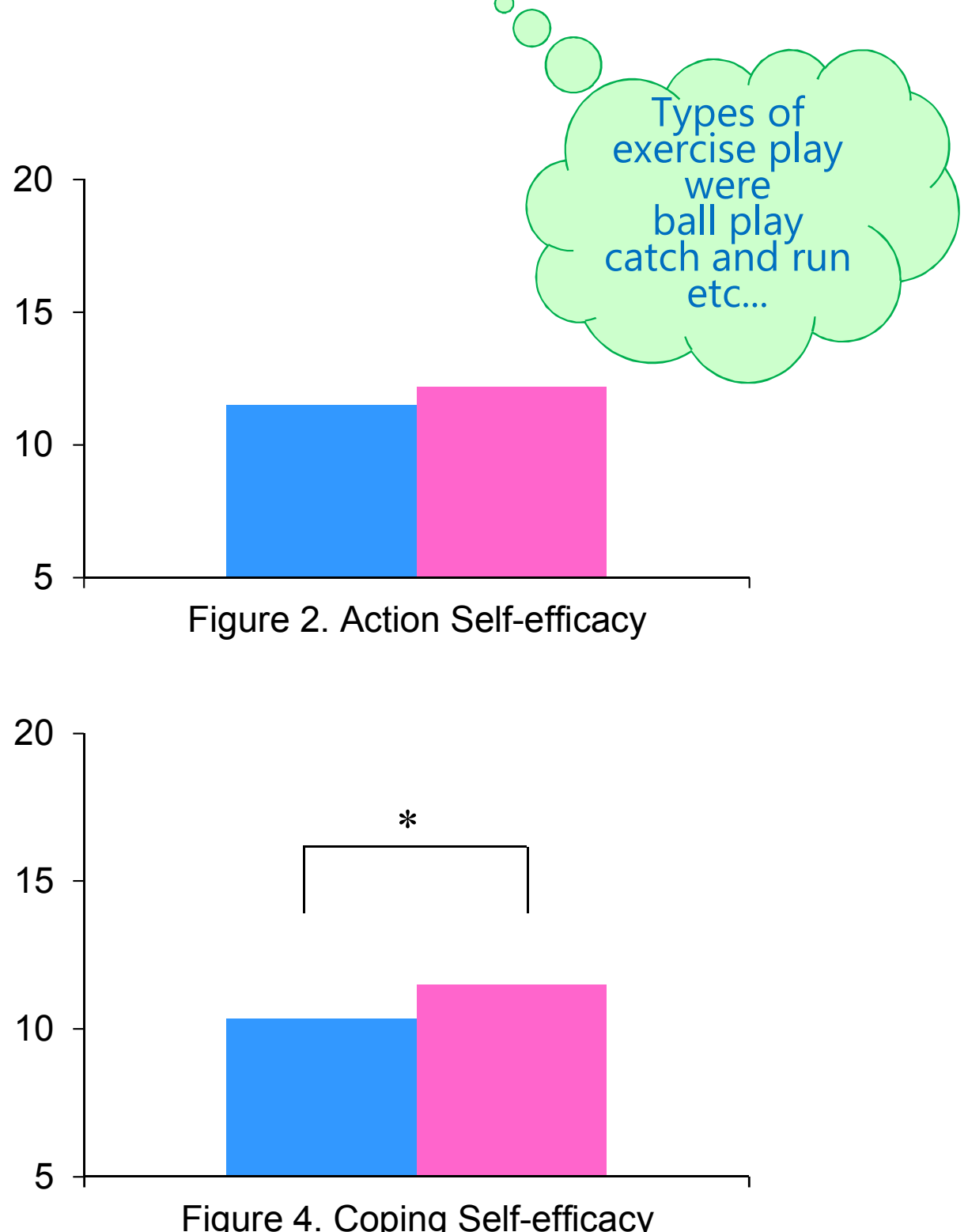

${ }^{* *} \mathrm{p}<.01,{ }^{*} \mathrm{p}<.05, \uparrow<.10$
The obtained results suggest that mothers who spend a longer time in exercise play with their children accomplish more general exercises and achieve higher exercise self-efficacy scores.

In particular, the conviction that exercise can be resumed even when exercising is interrupted, and the confidence of coping with barriers and implementing physical exercises are highly demonstrated in such mothers.

Future research should consider investigating the relationship between the exercise self-efficacy of children and exercise play with their mothers. 